Abanico Veterinario. Enero-Diciembre 2021; 11:1-12. http://dx.doi.org/10.21929/abavet2021.8 Artículo Original. Recibido: 31/08/2020. Aceptado: 20/01/2021. Publicado: 06/02/2021. Clave:2020-75.

\title{
Determinación de la calidad del semen criopreservado con lecitina de soya o yema de huevo, en machos cabríos
}

Determination of the quality of semen cryopreserved with soy lecithin or egg yolk, in male goats

\section{Moreno-Avalos Silvestre ${ }^{1 \text { ID }}$, Veliz-Deras Francisco ${ }^{\text {ID }}$, Calderon-Leyva Guadalupe $^{1 \text { ID }}$, Contreras-Villarreal Viridiana ID, Guillen-Muñoz Juan² ID, Angel- García Oscar 2 ID}

1Departamento de Producción Animal, Universidad Autónoma Agraria Antonio Narro. Torreón, Coahuila, México. ${ }^{2}$ Departamento de Ciencias Médico Veterinarias, Universidad Autónoma Agraria Antonio Narro. Torreón, Coahuila, México. *Autor responsable y de correspondencia: Ángel-García Oscar. Periférico y Carretera a Santa Fé SN, Colonia Valle Verde, Torreón, Coahuila, México, CP 27052. Correo. angelgarciao@hotmail.com, velizderas@gmail.com, gcalderon06@hotmil.com, dra.viridianac@gmail.com, mvz_guillen@hotmail.com

\section{RESUMEN}

El objetivo fue comparar la calidad del semen caprino criopreservado con diferentes tratamientos a base de lecitina de soya o yema de huevo. El semen fue colectado de machos cabríos Alpinos $(n=4)$, se utilizaron dos diluyentes comerciales: AndroMed® (1\% de lecitina de soya, LS); Optidyl® con 20\% (v/v) de Tris-yema de huevo; TY), y un tercer diluyente a base de citrato-yema de huevo (CY), en semen fresco (SF) y después fue enfriado de 37 a $4{ }^{\circ} \mathrm{C}$ durante $2 \mathrm{~h}$; semen refrigerado (SR), posteriormente se llenaron pajillas con semen y se congelaron en nitrógeno líquido a $-196{ }^{\circ} \mathrm{C}(\mathrm{SC})$. No existieron diferencias $(p>0.05)$ entre

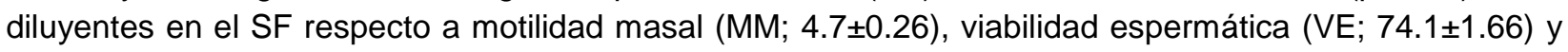
motilidad individual (Ml; 62.3 \pm 4.0$)$. En el mismo sentido, para el SR no existió diferencia $(p>0.05)$ entre diluyentes respecto a $\mathrm{MM}=3.83 \pm 0.4$, y $\mathrm{Ml}=52.1 \pm 6.0$, sin embargo, la VE varió $(\mathrm{p}<0.05)$ de acuerdo al diluyente, observando la menor viabilidad en LS vs. CY y TY (51.0 \pm 13.0 vs $71.3 \pm 3.0$ y $69.0 \pm 3.1)$. Respecto al SC, la MM, MI y VE favorecieron $(p<0.05)$ al diluyente TY vs. LS y CY $(2.4 \pm 0.5,32.5 \pm 8.3,41.3 \pm 13.0)$. Los resultados mostraron una mejor crio-preservación del semen caprino con el diluente Tris-yema respecto al de lecitina de soya.

Palabras clave: Lecitina de soya, diluyente, semen caprino

\begin{abstract}
The objective was to compare the quality of cryopreserved goat semen with soy lecithin or egg yolk. The semen was collected from male goats $(n=4)$, two commercial diluents AndroMed® (1\% soy lecithin, LS); Optidyl® $(20 \%$ (v/v) Tris-egg yolk; TY), and a citrate-egg yolk-based diluent (CY) were used in fresh semen (SF) and then cooled from 37 to $4{ }^{\circ} \mathrm{C}$ for $2 \mathrm{~h}$ (refrigerated semen, SR), afterwards straws were filled with semen and frozen in liquid nitrogen at $-196{ }^{\circ} \mathrm{C}(\mathrm{SC})$. There were no differences $(p>0.05)$ between diluents in the SF in the mass motility (MM; $4.7 \pm 0.26)$, sperm viability (VE; 74.1 \pm 1.66 ) and individual motility (MI; $62.3 \pm 4.0)$. In the same sense, for the SR there was no difference ( $p>0.05)$ between diluents with respect to MM (3.83 \pm 0.4$)$ and $\mathrm{MI}(52.1 \pm 6.0)$, however, the VE varied $(p<0.05)$ according to the diluent, observing the lowest viability in LS vs CY and TY (51.0 \pm 13.0 vs $71.3 \pm 3.0$ and $69.0 \pm 3.1)$. Regarding SC the MM, MI and VE obtained better values $(p<0.05)$ with the diluent TY vs LS and CY $(2.4 \pm 0.5,32.5 \pm 8.3,41.3 \pm 13.0)$. The results showed a better cryopreservation of goat semen with the diluent Tris-yolk compared to that of soy lecithin.
\end{abstract}

Keywords: Soy lecithin, diluent, goat semen 


\section{INTRODUCCIÓN}

Los diluyentes tradicionales que son agregados al semen para la preservación de la viabilidad y fertilidad de los espermatozoides durante la crioconservación incluyen yema de huevo (Lima-Verde et al., 2017); lo anterior, debido a que la yema de huevo protege al espermatozoide de los daños inducidos por la crioconservación durante el enfriamiento, congelación y descongelación al interactuar directamente con la membrana plasmática (Andrabi et al., 2008; Akçay et al., 2012; Sieme et al., 2016). Sin embargo, en los últimos años, frecuentemente se ha opinado en contra del uso de la yema de huevo debido a la gran variabilidad de sus componentes, lo que hace que la evaluación de sus beneficios sea compleja (Kulaksız et al., 2010); además se ha tratado de evitar el uso de diluyentes de origen animal, ya que podrían ser una posible ruta de transmisión de enfermedades (Lima-Verde et al., 2017; Ansari et al., 2017).

En lo que se refiere a la yema de huevo, algunos componentes indeseables (hormonas esteroides y sus moléculas precursoras) se consideran perjudiciales para la integridad del espermatozoide (Akhter et al., 2012; Lima-Verde et al., 2017). El componente principal de la yema de huevo que protege a la membrana espermática es la lipoproteína de baja densidad (LDL). Se ha demostrado que los fosfolípidos o lecitina de la yema de huevo hace a los espermatozoides menos sensibles al enfriamiento (Zeron et al., 2002). De manera particular, en el macho cabrío, existen interacciones negativas entre los fosfolípidos de la yema de huevo y la glándula bulbouretral, esta glándula secreta con el plasma seminal una enzima coagulante de la yema de huevo, la cual cataliza la hidrólisis de la lecitina de la yema de huevo en ácidos grasos y lisolecitina, que son citotóxicos (Ngoma et al., 2016). Por lo anterior, se han utilizado sustitutos de la yema de huevo químicamente definidos sin ser de origen animal (El-Sisy et al., 2018; Gamal et al., 2016), elaborados a base de lecitina de soya y que pueden ser alternativas potenciales para la criopreservación del semen (Akhter et al., 2012).

Se cree que los liposomas actúan de manera similar a las lecitinas de la yema de huevo o la leche (Belala et al., 2016). En el búfalo de agua, no se encontraron diferencias en términos de integridad acrosomal cuando se utilizaron diluyentes a base de yema de huevo, lecitina o liposomas de soya (Kumar et al., 2015; Singh et al., 2013). En este contexto, merecen atención especial los diluyentes elaborados en base a yema de huevo en cuanto a sus componentes y su efecto en comparación con los diluyentes basados en liposomas. Debido a que el efecto de los componentes mencionados anteriormente es poco conocido sobre la calidad del semen crioconservado en el macho cabrío, nos planteamos el objetivo de comparar los efectos de diluyentes a base de lecitina de soya o yema de huevo sobre la calidad del semen, conservado por refrigeración y congelación. 


\section{MATERIAL Y MÉTODOS}

\section{General}

Todos los métodos y manejo de las unidades experimentales utilizadas en este estudio fueron en estricto acuerdo con los lineamientos para el uso ético, cuidado y bienestar de animales en investigación a nivel internacional (FASS, 2010) y nivel nacional (NAM, 2002) con número de referencia de aprobación institucional UAAAN-UL con clave 38111425501002-2431.

\section{Localización y animales}

El experimento se realizó en el norte de México, en el Centro Caprino de la Universidad Autónoma Agraria Antonio Narro ( $26^{\circ}$ de Latitud Norte y $104^{\circ}$ de Longitud Oeste), durante la época reproductiva (enero). El área de estudio se encuentra a una altitud $1120 \mathrm{msnm}$, con una precipitación media anual de $230 \mathrm{~mm}$ y con temperatura promedio de $24{ }^{\circ} \mathrm{C}$, máxima de $41^{\circ} \mathrm{C}$ en mayo y junio, y mínima de $-1^{\circ} \mathrm{C}$ en diciembre y enero (CONAGUA, 2015). Se utilizaron machos cabríos adultos de la raza Alpino-francés ( $n=4$, de 1.5 a 2 años de edad), homogéneos en cuanto a peso vivo (PV; $75.0 \pm 0.32 \mathrm{~kg}$ ) y condición corporal (CC; $3.5 \pm 0.10$ unidades); con fertilidad probada previo al estudio experimental a través de evaluaciones frecuentes de calidad seminal. Durante el periodo experimental, los machos se alimentaron dos veces al día ( $800 \mathrm{~h}$ y $1800 \mathrm{~h}$ ), a libre acceso con una dieta basada en heno de alfalfa (18\% PC, $1.95 \mathrm{Mcal}$ de EM) y $100 \mathrm{~g}$ de concentrado comercial (21\% PC,1.7 Mcal EM) en base a sus requerimientos nutricionales (NRC, 2007). Los machos tuvieron libre acceso al agua limpia y sales minerales y un periodo de adaptación de 2 semanas previas a la investigación.

\section{Colección y procesamiento del semen}

El semen fue colectado por la mañana (800 a $1000 \mathrm{~h}$ ) cada $3 \mathrm{~d}$, durante tres semanas, se usó como estímulo para la extracción de semen una hembra en actividad estral. El semen fue recolectado con una vagina artificial estándar para ovinos y caprinos, mantenida a una temperatura de $38^{\circ} \mathrm{C}$, por lo que se precalentó a $42{ }^{\circ} \mathrm{C}$ previo a la recolección. Se colectaron un total de 24 eyaculados, después de cada extracción el semen fue sumergido inmediatamente en baño maría a $37^{\circ} \mathrm{C}$ para su posterior análisis macroscópico y microscópico durante los siguientes 10 minutos.

\section{Preparación de diluyentes y proceso de congelado}

De un total de 24 eyaculados ( 6 eyaculados por macho) y cada eyaculado se dividió en tres alícuotas en partes iguales para ser procesado para la criopreservación, utilizando dos diluyentes comerciales: AndroMed® (Minitübe, Tiefenbach, Alemania; con 1\% de lecitina de soya; LS); Optidyl® (CRYO-VET, Francia; con 20\% (v/v) de Tris-yema de huevo; TY), y un tercer diluyente a base de citrato-yema de huevo fresco (CY) obtenido de acuerdo a la técnica utilizada por Salamon y Maxwell, (2000). 
En el experimento fueron consideradas únicamente muestras con un volumen $>0.5 \mathrm{~mL}$, una concentración de $2.5 \times 10^{9} \mathrm{~mL}$, motilidad masal $\geq 3.0$ y viabilidad $\geq 70 \%$. Posteriormente las muestras ya diluidas fueron sometidas a 3 procesos para su evaluación: semen fresco (SF); semen refrigerado (SR, equilibrado a $4{ }^{\circ} \mathrm{C}$ por 2 horas); y semen congelado (SC). Después de refrigerar el semen se llenaron las pajillas de 0.25 $\mathrm{mL}$ y para el proceso de congelación se colocaron a $7 \mathrm{~cm}$ de nitrógeno líquido ( $\mathrm{NL}$, $\left.140^{\circ} \mathrm{C}\right)$ por $10 \mathrm{~min}$; después se sumergieron directamente en el $\mathrm{NL}\left(-196^{\circ} \mathrm{C}\right)$ y se almacenaron hasta su análisis (Jerez et al., 2016). En cada uno de los estados de conservación (SF, SR y SC), el semen se analizó, inmediatamente y cada 15 minutos durante el proceso de conservación, para evaluar la motilidad masal e individual y la viabilidad. En el caso del SC, éste se analizó 24 horas después, para lo cual, la pajilla se descongeló, sumergiéndola en agua atemperada $\left(37^{\circ} \mathrm{C}\right)$ durante $30 \mathrm{~s}$.

\section{Variables evaluadas}

La motilidad masal (MM; \%), se evaluó con el uso de una plataforma precalentada (37 $\left.{ }^{\circ} \mathrm{C}\right)$, colocando una gota de semen puro $(20 \mu \mathrm{l})$ sobre una lámina portaobjetos en el microscopio óptico con objetivo de 10x, y de acuerdo con el movimiento observado se asignó un puntaje de escala arbitraria de 1 a 5 , donde $1=25 \%$ y $5=100 \%$ de espermatozoides móviles (Mahsud et al., 2013). La motilidad individual (Ml; \%), se determinó en base a la proporción de espermatozoides progresivamente móviles, para ello, se colocó una gota $(10 \mu \mathrm{L})$ de semen sobre una lámina portaobjetos y fue cubierta con una laminilla cubreobjetos; posteriormente se observó al microscopio con objetivo de 40x. La viabilidad espermática (VE; \%), se evaluó mediante el uso de la técnica de tinción con eosina-nigrosina (Kafi et al., 2004), se observaron al menos 200 espermatozoides por muestra mediante microscopio óptico, utilizando el objetivo de 100X, y se calculó el porcentaje de células vivas (sin teñir) y de células muertas (teñidas de color rosa). Todas las evaluaciones fueron realizadas siempre por el mismo evaluador calificado.

\section{Análisis estadístico}

Los datos fueron analizados mediante un ANOVA usando el procedimiento Modelo Lineal General (GLM). Las medias obtenidas de los parámetros seminales fueron comparadas usando una prueba de $t$. Se contempló el efecto del uso de los diferentes diluyentes, los estados del proceso de crioconservación y la interacción de estos. Todos los datos fueron analizados utilizando el paquete estadístico SAS V9.1 (SAS, 2005). Las diferencias fueron consideradas significativas a un valor de $P \leq 0.05$.

\section{RESULTADOS Y DISCUSIÓN}

Los resultados de los diferentes parámetros evaluados para determinar la calidad del semen diluido con LS, TY o $\mathrm{CY}$, en semen fresco (SF), refrigerado durante $2 \mathrm{~h}(\mathrm{SR})$ y semen congelado (SC) se resumen en el cuadro 1. 
Cuadro 1. Medias ( $\pm E E M)$ para la calidad del semen criopreservado de machos cabríos Alpinosfrances diluido con lecitina de soya o yema de huevo

\begin{tabular}{lccc}
\hline \multicolumn{1}{c}{ Parámetros } & $\begin{array}{c}\text { MM } \\
\text { (escala, } 1-5)\end{array}$ & $\begin{array}{c}\text { Ml } \\
(\%)\end{array}$ & $\begin{array}{c}\text { VE } \\
(\%)\end{array}$ \\
\hline Semen Fresco & & & $76 \pm 0.2^{\mathrm{a}}$ \\
\hline LS & $4.8 \pm 0.3^{\mathrm{a}}$ & $63.0 \pm 1.2^{\mathrm{a}}$ & $75.0 \pm 2.0^{\mathrm{a}}$ \\
TY & $4.8 \pm 0.3^{\mathrm{a}}$ & $62.5 \pm 1.4^{\mathrm{a}}$ & $71.3 \pm 4.0^{\mathrm{a}}$ \\
CY & & $61.3 \pm 4.3^{\mathrm{a}}$ & \\
\hline Semen Refrigerado & $3.1 \pm 1.0^{\mathrm{a}}$ & $44.0 \pm 12.0^{\mathrm{ab}}$ & $51.0 \pm 13.0^{\mathrm{bc}}$ \\
\hline LS & $4.4 \pm 0.1^{\mathrm{a}}$ & $57.5 \pm 2.5^{\mathrm{a}}$ & $71.3 \pm 2.4^{\mathrm{a}}$ \\
TY & $4.0 \pm 0.2^{\mathrm{a}}$ & $55.0 \pm 3.5^{\mathrm{a}}$ & $69.0 \pm 3.1^{\mathrm{ab}}$ \\
CY & & & \\
\hline Semen Congelado & $1.0 \pm 0.4^{\mathrm{c}}$ & $11.0 \pm 6.4^{\mathrm{c}}$ & $11.0 \pm 7.1^{\mathrm{d}}$ \\
\hline LS & $2.4 \pm 0.5^{\mathrm{b}}$ & $32.5 \pm 8.3^{\mathrm{b}}$ & $41.3 \pm 13.0^{\mathrm{c}}$ \\
TY & $0.5 \pm 0.3^{\mathrm{c}}$ & $6.3 \pm 3.8^{\mathrm{c}}$ & $2.5 \pm 2.5^{\mathrm{d}}$ \\
\hline CY & & & \\
\hline
\end{tabular}

Tratamientos: Andromed® (LS; $1 \%$ de lecitina de soya), Optidyl® (TY: Tris- $20 \%$ de yema de huevo) 0 citrato- yema de huevo (CY). Motilidad masal (MM; escala,1-5), Motilidad individual (MI; \%), Viabilidad espermatica (VE; \%). abcd Superíndices desiguales entre filas indican diferencia estadística significativa $(\mathrm{P} \leq 0.05)$.

En el SF, se obtuvieron valores similares entre los grupos $(P>0.05)$ en cada una de las variables evaluadas [MM $(4.7 \pm 0.26 \%)$, VE $(74.1 \pm 1.66 \%)$ y Ml $(62.3 \pm 4.0 \%)$ ]. Los resultados sugieren que la composición de los diluyentes utilizados en este estudio afecta la calidad de los espermatozoides posterior al proceso de descongelamiento. Sin embargo, la calidad seminal post descongelación se vio más afectada cuando se utilizó el diluyente a base LS, en comparación con el TY. Estos resultados son similares a los reportados en equinos y cérvidos; en los cuales se muestra una mayor calidad seminal cuando se utilizan diluyentes a base de yema de huevo (Pillet et al., 2012; Stewart et al., 2018). Es probable que los resultados encontrados se deban a que el componente Trisyema de huevo ayuda a disminuir la generación de especies reactivas de oxígeno (ROS) manteniendo así el potencial de integridad de la membrana al reducir el choque térmico causado por los cambios de temperatura en el proceso de crioconservación (Alcay et al., 2016; Seifi-Jamadi et al., 2017). Lo anterior puede estar relacionado con el componente efectivo de la yema de huevo que es la lipoproteína de baja densidad (20\%), que contiene 
el TY y funciona como fracción crioprotectora que ayuda a mantener el semen de mejor calidad (Amirat et al., 2004; Forouzanfar et al., 2010; Alcay et al., 2016).

Al comparar los efectos de los diluyentes en las condiciones de SR, no existieron diferencias $(P>0.05)$ en la MM y Ml $(3.83 \pm 0.4 \%$ y $52.1 \pm 6.0 \%$, respectivamente); sin embargo, el porcentaje de VE fue más bajo con el diluyente a base de LS comparado con el TY y CY $(51.0 \pm 13.0$ vs $71.3 \pm 3.0$ y $69.0 \pm 3.1$ respectivamente; $P<0.05)$.

Los resultados reportados en el presente experimento referentes al diluyente TY concuerdan con lo reportado por Celeghini et al. (2008), que indican una mayor integridad del acrosoma de espermatozoides de toro, y Konyak et al. (2018) quienes encontraron que después del equilibrio y la congelación del semen de machos cabríos, la motilidad espermática es significativamente mayor al utilizar el diluyente Tris con $20 \%$ de yema de huevo, respecto al conformado por $1 \%$ de lecitina de soya. Al respecto, se ha comprobado que la lecitina de soya no es capaz de evitar la peroxidación lipídica que ocurre durante el proceso de enfriamiento del espermatozoide (Salmani et al., 2013).

Previas investigaciones en ovinos han demostrado que el semen diluido con lecitina de soya presenta daño en la membrana espermática, y en consecuencia daño a nivel mitocondrial, lo que resulta en una menor movilidad y fertilidad de los espermatozoides (Del Valle et al., 2012; Lima-Verde et al., 2017). Konyak et al. (2018) atribuye la baja en la calidad espermática del semen expuesto a la LS a diferencias en la concentración de la lecitina de soya utilizada, en relación a esto, Forouzanfar et al. (2010) observaron en semen de carneros que los diluyentes que contenían concentraciones de $1 \%$ de lecitina presentaban mayor viabilidad espermática, comparado con los de $2 \%$ de lecitina, y además que el rango 1 a $1.5 \%$ de lecitina de soya en el diluyente mostraron mejores características del semen después de la preservación.

En el SC, los valores de MM, MI y VE fueron superiores en el semen diluido con TY $(2.4 \pm 0.5,32.5 \pm 8.3,41.3 \pm 13.0$, respectivamente; $\mathrm{P} \leq 0.05)$ en comparación con el semen diluido con LS y el CY que disminuyeron su MM, Ml y VE drásticamente (1.0.0 \pm 0.4 , $11.0 \pm 6.4,11.0 \pm 7.1$ y $0.5 \pm 0.3 \pm, 6.3 \pm 3.8$ y $2.5 \pm 2.5$ respectivamente; $P \leq 0.05$ ). De igual forma los resultados en nuestro estudio en el CY post-descongelación fue inferiores en comparación con el TY. Es probable que estos resultados estén asociados a los componentes del diluyente, específicamente al porcentaje de yema de huevo, en el diluyente CY fue a una concentración del $15 \%$, y en el TY al $20 \%$. Estos resultados concuerdan con Fourouzanfar et al. (2010), quienes reportan que la motilidad y viabilidad espermática post-descongelación son superiores cuando se utiliza una concentración de yema de huevo al $20 \%$ que cuando se utiliza al $15 \%$. Del mismo modo, otros trabajos confirman que el aumento en la concentración de la yema de huevo mejora la preservación de la calidad espermática (Amirat et al., 2004; Forouzanfar et al., 2010; Alcay et al., 2016). La razón de este mejoramiento puede ser debido a que los fosfolípidos que contiene la yema de huevo como la fosfatidilcolina, son importantes para el 
mantenimiento de la integridad de la membrana espermática durante el proceso de congelación y post-descongelación (Mousa et al., 2002; Amirat et al., 2004; Forouzanfar et al., 2010; Alcay et al., 2016). Por lo que es probable que un alto porcentaje de yema de huevo mejore la viabilidad espermática observada en el TY, y ésta contribuyera a mantener los niveles de ácidos grasos polinsatrurados necesarios para la membrana de los espermatozoides, siendo menos susceptibles a la peroxidación lipídica destructiva (Cerolini et al., 2001; Kaeoket et al., 2010).

Por otra parte, la mala calidad seminal post-descongelación del CY, pudo deberse a que la yema de huevo tiene alto riesgo de sufrir una contaminación microbiana, lo cual pudo disminuir la calidad seminal, post-descongelación (Aboagla et al., 2004; Kulakzis et al., 2010); y al ser la yema de huevo comercial (CY), pudo no tener una buena calidad sanitaria, lo que perjudicó la calidad seminal post-congelación. Otro factor que pudo afectar la calidad del semen del CY, es la dieta y el manejo de las aves productoras (LimaVerde et al., 2017). En efecto, varios estudios han mostrado que las yemas de huevo de diferentes especies de aves muestran una variación en sus componentes, lo que resulta en diferentes efectos en el proceso de criopreservación sobre los espermatozoides (Trimeche et al., 1997; Bathgate et al., 2006; Singh et al., 2013). Además, la yema de huevo puede contener metabolitos dañinos y endotoxinas que afectan la viabilidad de los espermatozoides (Vidal et al., 2013), así como hormonas esteroides que reducen la motilidad espermática (El-Sisy et al., 2018). Previos estudios de laboratorio revelan que, mediante eliminación de algunos componentes en la yema de huevo por centrifugación; además de ciertas sustancias en la yema que inhiben la respiración y la motilidad espermática, sugiriendo el reemplazo la yema de huevo entera por la fracción crioprotectora (Amirat et al., 2004).

\section{CONCLUSIONES}

Los resultados del presente estudio no mostraron diferencias estadísticas significativas entre el uso de los diferentes diluyentes para la conservación del SF y SR; sin embargo, el Tris-yema de huevo obtuvo una mayor motilidad masal e individual y viabilidad postdescongelación en comparación con el diluyente a base de lecitina de soya durante el proceso de crioconservación del semen de machos cabríos Alpinos

\section{AGRADECIMIENTOS}

Los autores agradecen el apoyo financiero brindado a la Secretaria de Agricultura y Desarrollo Rural y al Consejo Nacional de Ciencia y Tecnología (SADER-CONACYT, México) por el apoyo financiero otorgado a través del Fondo Sectorial de Investigación en Materia Agrícola, Pecuaria, Acuacultura, Agrobiotecnología y Recursos Fitogeneticos, 2017-04-291691. 


\section{LITERATURA CITADA}

ABOAGLA EME, Terada T. 2004. Effects of egg yolk during the freezing step of cryopreservation on the viability of goat spermatozoa. Theriogenology. 62(6):1160-1172. ISSN: 0093-691X. https://doi.org/10.1016/j.theriogenology.2004.01.013

AKÇAY E Kulaksız R, Daşkin A, Çebi Ç, Tekin K. 2012. The effect of different dilution rates on post-thaw quality of ram semen frozen in two different egg-yolk free extenders. Slovenian Veterinary Research. 49 (2):97-102. ISSN: 1580-4003. https://www.academia.edu/24162140/The_Effect_of_Different_Dilution_Rates_on_Post_ Thaw_Quality_of_Ram_Semen_Frozen_in_Two_Different_Eggyolk_Free_Extenders

AKHTER S, Ansari MS, Andrabi SMH, Rakha BA, Ullah N, Khalid M. 2012. Soya-lecithin in extender improves the freezability and fertility of buffalo (Bubalus bubalis) bull spermatozoa. Reproduction in Domestic Animals. 47(5):815-819.ISSN: 1439-0531.x https://doi.org/10.1111/j.1439-0531.2011.01973.x

ALCAY S, Gokce E, Toker MB, Onder NT, Ustuner B, Uzabacı E, Cavus S. 2016. Freezedried egg yolk based extenders containing various antioxidants improve post-thawing quality and incubation resilience of goat spermatozoa. Cryobiology. 72(3): 269-273. ISSN: 0011-2240. https://doi.org/10.1016/j.cryobiol.2016.03.007

AMIRAT L, Tainturier D, Jeanneau L, Thorin C, Gérard O, Courtens JL, Anton M. 2004. Bull semen in vitro fertility after cryopreservation using egg yolk LDL: a comparison with Optidy ${ }^{\circledR}$, a commercial egg yolk extender. Theriogenology. 61(5): 895-907. ISSN: 0093691X. https://doi.org/10.1016/S0093-691X(03)00259-0

ANDRABI SMH, Ansari MS, Ullah N, Anwar M, Mehmood A, Akhter S. 2008. Duck egg yolk in extender improves the freezability of buffalo bull spermatozoa. Animal Reproduction Science. 104(2-4): 427-433. ISSN: 0378-4320. https://doi.org/10.1016/j.anireprosci.2007.07.003

ANSARI MS, Rakha BA, Akhter S. 2017. Cryopreservation of Nili-Ravi buffalo (Bubalus bubalis) semen in AndroMed® extender; in vitro and in vivo evaluation. Reproduction in Domestic Animals. 52(6):992-997. ISSN: 1439-0531. https://doi.org/10.1111/rda.13008

BATHGATE R, Maxwell WMC, Evans G. 2006. Studies on the effect of supplementing boar semen cryopreservation media with different avian egg yolk types on in vitro postthaw sperm quality. Reproduction in Domestic Animals. 41(1):68-73. ISSN: 1439-0531. https://doi.org/10.1111/j.1439-0531.2006.00623.x 
BELALA R, Briand-Amirat L, Vinciguerra L, Tainturier D, Kaidi R, Thorin C, Bencharif D. 2016. Effect of equilibration time on the motility and functional integrity of canine spermatozoa frozen in three different extenders. Research in Veterinary Science. 106: 6673. ISSN:0034-5288. https://doi.org/10.1016/j.rvsc.2016.03.010

CELEGHINI ECC, de Arruda RP, de Andrade AFC, Nascimento J, Raphael CF, Rodrigues PHM. 2008. Effects that bovine sperm cryopreservation using two different extenders has on sperm membranes and chromatin. Animal Reproduction Science. 104(2-4):119-131. ISSN:0378-4320. https://doi.org/10.1016/j.anireprosci.2007.02.001

CEROLINI S, Maldjian, A, Pizzi F, Gliozzi T. M. 2001. Changes in sperm quality and lipid composition during cryopreservation of boar semen. Reproduction. 121(3):395-401. ISSN: 1741-7899. https://doi.org/10.1530/rep.0.1210395

CONAGUA. 2015. Normales climatológicas por estación. Ciudad de México: Servicio Meteorológico Nacional, Comisión Nacional del Agua. https://smn.conagua.gob.mx/es/

DEL VALLE I, Gómez-Durán A, Holt WV, Muiño-Blanco T, Cebrián-Pérez JA. 2012. Soy lecithin interferes with mitochondrial function in frozen-thawed ram spermatozoa. Journal of Andrology. 33(4):717-725. ISSN: https://doi.org/10.2164/jandrol.111.014944

EL-SISY GA, El-Badry DA, El-Sheshtawy RI, El-Nattat WS. 2018. Effects of Phoenix dactylifera pollen grains extract supplementation on post-thaw quality of Arabian stallion semen. Bulgarian Journal of Veterinary Medicine. 21(1):40-49. ISSN: 1.311-1477. https://doi.org/10.15547/bjvm.1044

FASS. 2010. Guide for the Care and Use of Agricultural Animals in Agricultural Research and Teaching, 3rd ed. Federation Animal Science Society, Savoy, IL, USA. ISBN: 978956-14-2161-5.

https://books.google.com.mx/books?id=EA1QDwAAQBAJ\&printsec=frontcover\&dq=Gui $\mathrm{de}+$ for+the+Care+and+Use+of+Agricultural+Animals+in+Agricultural+Research+and+Te aching\&hl=es\&sa=X\&ved=2ahUKEwi8svisttjrAhUIW60KHUttBHMQ6AEwAnoECAUQAg \#v=onepage\&q=Guide\%20for\%20the\%20Care\%20and\%20Use\%20of\%20Agricultural\% 20Animals\%20in\%20Agricultural\%20Research\%20and\%20Teaching\&f=false

FOROUZANFAR M, Sharafi M, Hosseini S M, Ostadhosseini S, Hajian M, Hosseini L, Nasr-Esfahani MH. 2010. In vitro comparison of egg yolk-based and soybean lecithinbased extenders for cryopreservation of ram semen. Theriogenology. 73(4):480-487. ISSN: 0093-691X. https://doi.org/10.1016/j.theriogenology.2009.10.005 
GAMAL A, El-Maaty AMA, Rawash ZM. 2016. Comparative blood and seminal plasma oxidant/antioxidant status of Arab stallions with different ages and their relation to semen quality. Asian Pacific Journal of Reproduction. 5(5):428-433. ISSN: 2305-0500. https://doi.org/10.1016/j.apjr.2016.07.006

JEREZ R, González N, Olaciregui M, Luño V, de Blas I, Gil L. 2016. Use of soy milk combined with different cryoprotectants for the ram semen cryopreservation. Small Ruminant Research. 134:34-38. ISNN: 0921-4488. https://doi.org/10.1016/j.smallrumres.2015.12.003

KAEOKET K, Chanapiwat $P$, Tummaruk $P$, Techakumphu M. 2010. Supplemental effect of varying L-cysteine concentrations on the quality of cryopreserved boar semen. Asian Journal of Andrology. 12(5):760. https://dx.doi.org/10.1038/aja.2010.48

KAFI M, Safdarian M, Hashemi M. 2004. Seasonal variation in semen characteristics, scrotal circumference, and libido of Persian Karakul rams. Small Ruminant Research. 53 (1-2):133-139. ISSN: 0921-4488. https://doi.org/10.1016/j.smallrumres.2003.07.007

KONYAK P, Mandal A, Mondal M, Bhakat C, Das SK, Rai S, Karunakaran M. 2018. Preservation of black Bengal buck semen in soybean lecithin based chemically defined extender. Indian Journal of Animal Research. 52(8):1151-1154. ISSN: 0976-0555. https://doi.org/10.18805/ijar.B-3335

KULAKSIZ R, Çebi Ç, Akçay E, Daşkın A. 2010. The protective effect of egg yolk from different avian species during the cryopreservation of Karayaka ram semen. Small Ruminant Research. 88(1):12-15. ISSN: 0921-4488. https://doi.org/10.1016/j.smallrumres.2009.11.014

KUMAR P, Saini M, Kumar D, Balhara AK, Yadav SP, Singh P, Yadav PS. 2015. Liposome-based semen extender is suitable alternative to egg yolk-based extender for cryopreservation of buffalo (Bubalus bubalis) semen. Animal Reproduction Science. 159:38-45. ISSN: 0378-4320. https://doi.org/10.1016/j.anireprosci.2015.05.010

LIMA-VERDE IB, Johannisson A, Ntallaris T, Al-Essawe E, Al-Kass Z, Nongbua T, Morrell, JM. 2017. Effect of freezing bull semen in two non-egg yolk extenders on post-thaw sperm quality. Reproduction in Domestic Animals. 53(1): 127-136. ISSN: 1439-0531 https://doi.org/10.1111/rda.13080

MAHSUD T, Jamil H, Qureshi ZI, Asi MN, Lodhi LA, Waqas MS, Ahmad A. 2013. Semen quality parameters and selected bio-chemical constituents level in plasma of Lohi rams. Small Ruminant Research. 113(1): 175-178. ISSN: 0921-4488. https://doi.org/10.1016/j.smallrumres.2013.04.004 
MOUSSA M, Martinet V, Trimeche A, Tainturier D, Anton M. 2002. Low density lipoproteins extracted from hen egg yolk by an easy method: cryoprotective effect on frozen-thawed bull semen. Theriogenology. 57(6):1695-1706. ISSN: 0093-691X. https://doi.org/10.1016/S0093-691X(02)00682-9

NAM. 2002. Guide for the Care and Use of Laboratory Animals. Co-produced by the National Academy of Medicine-Mexico and the Association for Assessment and Accreditation of Laboratory Animal Care International, 1st ed. Harlan Mexico, DF, Mexico. ISBN: 978-0-309-15400-0.

https://books.google.com.mx/books?id=GyxkAgAAQBAJ\&printsec=frontcover\&dq=Guid e+for+the+Care+and+Use+of+Laboratory+Animals. + ISBN\&hl=es\&sa=X\&ved=2ahUKEw jr1pGOtNjrAhUEPq0KHRA3AU0Q6AEwAHoECAIQAg\#v=onepage\&q=Guide\%20for\%2 0the $\% 20$ Care\%20and\%20Use\%20 \%20Laboratory\%20Animals.\%20ISBN\&f=false

NGOMA L, Kambulu L, Mwanza M. 2016. Factors Influencing goat's semen fertility and storage: A Literature Review. Journal of Human Ecology. 56(1-2):114-125. ISSN: 24566608. https://doi.org/10.1080/09709274.2016.11907045

NRC. 2007. Nutrient Requirements of Small Ruminants: Sheep, Goats, Cervids and New World Camelids. National Research Council, National Academies Press, Washington, USA. ISBN: 978-0-309-47323-1. https://www.nap.edu/catalog/11654/nutrientrequirements-of-small-ruminants-sheep-goats-cervids-and-new

PILLET E, Labbe C, Batellier F, Duchamp G, Beaumal V, Anton M, Magistrini M. 2012. Liposomes as an alternative to egg yolk in stallion freezing extender. Theriogenology. 77(2):268-279. ISSN: 0093-691X. https://doi.org/10.1016/j.theriogenology.2011.08.001

SALAMON S, Maxwell W MC. 2000. Storage of ram semen. Animal Reproduction Science. 62 (1-3):77-111. ISSN: 0378-4320. https://doi.org/10.1016/S03784320(00)00155-X

SALMANI H, Nabi MM, Vaseghi-Dodaran $\mathrm{H}$, Rahman MB, Mohammadi-Sangcheshmeh A, Shakeri M, Zhandi M. 2013. Effect of glutathione in soybean lecithin-based semen extender on goat semen quality after freeze-thawing. Small Ruminant Research.112(13):123-127. ISSN:0921-4488. https://doi.org/10.1016/j.smallrumres.2012.12.015

SAS (Statistical Analysis System) 2005. Statistical Analysis Software SAS/STAT®. version 9.1, Cary, N.C., USA: SAS Institute Inc.

SEIFI-JAMADI A, Ahmad E, Ansari M, Kohram, H. 2017. Antioxidant effect of quercetin in an extender containing DMA or glycerol on freezing capacity of goat semen. Cryobiology. 75:15-20. ISSN: 0011-2240. https://doi.org/10.1016/j.cryobiol.2017.03.002 
SIEME H, Oldenhof H, Wolkers WF. 2016. Mode of action of cryoprotectants for sperm preservation. Animal Reproduction Science. 169:2-5. ISSN: 0378-4320. https://doi.org/10.1016/j.anireprosci.2016.02.004

SINGH MAHAK, Ramteke SS, Ghosh SK, Prasad JK, Rajoriya JS. 2013. Efficacy of egg yolk from three avian species on semen freezability of Tharparkar bull. Indian Journal Animals Reproduction. 34(2):25-28. ISSN: 0970 - 2997. https://scholar.google.com/citations?user=ot596g4AAAAJ\&hl=en\#d=gs_md_citad\&u=\%2Fcitations\%3Fview_op\%3Dview_citation\%26hl\%3Den\%26user\%3Dot596g4AA AAJ\%26citation_for_view\%3Dot596g4AAAAJ\%3AWF5omc3nYNoC\%26tzom\%3D300

STEWART JL, Shipley CF, Ellerbrock RE, Schmidt L, Lima FS, Canisso IF. 2018. Physiological variations in reproductive and metabolic features of white-tailed deer (Odocoileus virginianus) bucks throughout the rutting season. Theriogenology. 114(1):308-316. ISSN: 0093-691X. https://doi.org/10.1016/j.theriogenology.2018.04.015

TRIMECHE A, Anton M, Renard P, Gandemer G, Tainturier D. 1997. Quail egg yolk: a novel cryoprotectant for the freeze preservation of Poitou jackass sperm. Cryobiology. 34(4):385-393. ISSN: 0011-2240. https://doi.org/10.1006/cryo.1997.2009

VIDAL AH, Batista AM, da Silva ECB, Gomes WA, Pelinca MA, Silva SV, Guerra MMP. 2013. Soybean lecithin-based extender as an alternative for goat sperm cryopreservation. Small Ruminant Research. 109(1):47-51. ISSN: 0921-4488. https://doi.org/10.1016/j.smallrumres.2012.07.022

ZERON Y, Tomczak M, Crowe J, Arav A. 2002. The effect of liposomes on thermotropic membrane phase transitions of bovine spermatozoa and oocytes: implications for reducing chilling sensitivity. Cryobiology. 45(2):143-152. ISSN: 0011-2240. https://doi.org/10.1016/S0011-2240(02)00123-2 